\title{
An improved vacuum formulation for 2D finite-difference modeling of Rayleigh waves including surface topography and internal discontinuities
}

\author{
Chong Zeng ${ }^{1}$, Jianghai $\mathrm{Xia}^{2}$, Richard D. Miller ${ }^{3}$, and Georgios P. Tsoflias ${ }^{4}$
}

\begin{abstract}
Rayleigh waves are generated along the free surface and their propagation can be strongly influenced by surface topography. Modeling of Rayleigh waves in the near surface in the presence of topography is fundamental to the study of surface waves in environmental and engineering geophysics. For simulation of Rayleigh waves, the traction-free boundary condition needs to be satisfied on the free surface. A vacuum formulation naturally incorporates surface topography in finite-difference (FD) modeling by treating the surface grid nodes as the internal grid nodes. However, the conventional vacuum formulation does not completely fulfill the free-surface boundary condition and becomes unstable for modeling using high-order FD operators. We developed
\end{abstract}

a stable vacuum formulation that fully satisfies the free-surface boundary condition by choosing an appropriate combination of the staggered-grid form and a parameter-averaging scheme. The elastic parameters on the topographic free surface are updated with exactly the same treatment as internal grid nodes. The improved vacuum formulation can accurately and stably simulate Rayleigh waves along the topographic surface for homogeneous and heterogeneous elastic models with high Poisson's ratios $(>0.4)$. This method requires fewer grid points per wavelength than the stress-image-based methods. Internal discontinuities in a model can be handled without modification of the algorithm. Only minor changes are required to implement the improved vacuum formulation in existing 2D FD modeling codes.

\section{INTRODUCTION}

Dispersive Rayleigh waves have been widely employed to estimate S-wave velocities in shallow layers (Nazarian and Stokoe, 1984; Xia et al., 1999, 2003, 2004, 2006; Calderón-Macías and Luke, 2007; Luo et al., 2009a; Socco et al., 2010). Numerical modeling of Rayleigh waves has been investigated in near-surface seismology for various purposes including a study of attenuation (Carcione, 1992) and a shallow cavity investigation (Gelis et al., 2005). Rayleigh waves are the combination of $P$-waves and the vertical component of shear waves (SV-waves) along the free surface. They can be simulated in the 2D P-SV wave domain by solving the vector wave equation through numerical methods (e.g., the finite-difference [FD] method). The physical discontinuity of the earth's surface poses constraints on the elastic wave solutions. A vacuum-earth interface is a traction-free surface on which the free-surface boundary condition is satisfied (Aki and Richards, 2002). On a horizontal vacuum-earth plane in the 3D Cartesian coordinate system, the shear stress components and the vertical normal stress are all zero. Numerical implementation of this freesurface condition is critical for the accuracy of simulated Rayleigh waves. The surface topography strongly distorts the near-surface wavefield. An appropriate implementation of the free surface that includes topography is key to the accurate simulation of Rayleighwave propagation in near-surface.

In the FD method, the earth model is usually discretized into rectangular or cubical cells. The conventional FD implementation of the free-surface boundary condition is only valid for the

\footnotetext{
Manuscript received by the Editor 15 February 2011; revised manuscript received 25 July 2011; published online 6 February 2012.

${ }^{1}$ Presently BGP International Inc., Research \& Development Center, Houston, Texas, USA; formerly University of Kansas, Kansas Geological Survey, Lawrence, Kansas, USA. E-mail: zengchong@gmail.com.

${ }^{2}$ Presently The China University of Geosciences, Subsurface Imaging and Sensing Laboratory, Institute of Geophysics and Geomatics, Wuhan, Hubei, China; formerly University of Kansas, Kansas Geological Survey, Lawrence, Kansas, USA. E-mail: jxia@kgs.ku.edu.

${ }^{3}$ University of Kansas, Kansas Geological Survey, Lawrence, Kansas, USA. E-mail: rmiller@kgs.ku.edu.

${ }^{4}$ University of Kansas, Department of Geology, Lawrence, Kansas, USA. E-mail: tsoflias@ku.edu.

(C) 2012 Society of Exploration Geophysicists. All rights reserved.
} 
horizontal (planar) earth surface (Mittet, 2002; Xu et al., 2007). The staggered-grid technique (Virieux, 1986) and the parameteraveraging scheme (Moczo, 2002) increase the implementation complexity of the free-surface boundary condition in the presence of surface topography.

Jih et al. (1988) introduce a technique to decompose an irregular free surface into line segments to handle surface topography. Tessmer et al. (1992) propose a coordinate mapping method including surface topography. Robertsson (1996) analyzes the categories of surface grid nodes and presents a numerical free-surface boundary condition with an arbitrary topography. Robertsson's method can be considered an extension of the classical stress-image technique originally proposed for the horizontal free surface by Levander (1988). This technique approximates the topographic earth surface by a fine-grid staircase shape. The stress-image technique is used to update the particle velocities for grid nodes located on the free surface. For grid nodes above the free-surface, the particle velocities are forced to be zero.

In the image method (Robertsson, 1996), grid nodes on the free surface are classified into seven categories for a 2D earth model. Each category employs a different strategy to update the stress and velocity components. The classification of surface grid nodes can be complicated for an arbitrary surface topography and is challenging when the model contains internal discontinuities (such as the tunnel earth model commonly investigated in near-surface seismology [Gelis et al., 2005; Xia et al., 2007a]). Moreover, the accuracy of the image method for Rayleigh waves is reduced along the surface topography and the simulation requires more grid points per wavelength (ppw) than conventional seismic modeling focusing on P-waves (Robertsson, 1996).

Another approach to incorporating surface topography is using the so-called vacuum formulation (Zahradník et al., 1993; Graves, 1996), in which the physical parameters are set to zero on the grid nodes above the free surface. The free-surface boundary is then treated as an internal interface inside the model. Within this method, the surface topography and internal discontinuities are automatically identified by data variations of elastic coefficients. Parameters for all grid nodes throughout the model are updated in exactly the same manner, which simplifies program implementation. Numerical tests indicated that the simple vacuum formulation is only stable for second-order spatial FD operators (Graves, 1996). Moreover, the conventional vacuum formulation does not completely fulfill

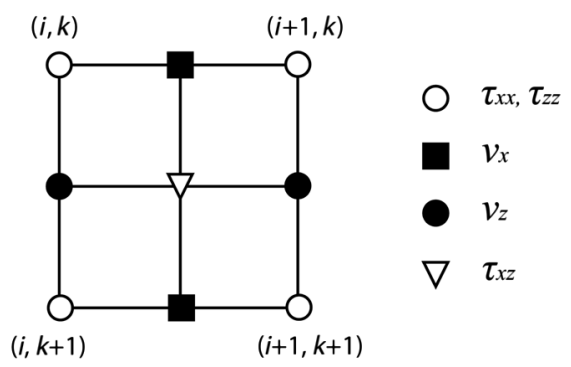

Figure 1. The staggered-grid scheme used for the proposed vacuum formulation. The light circles are the grid nodes. The grid position is described by the indices $i$ and $k$. The normal stress tensor components $\tau_{x x}$ and $\tau_{z z}$, Lamé coefficients $\lambda$ and $\mu$, and the mass density $\rho$ are all defined at the grid nodes. The triangle is the shear stress tensor component $\left(\tau_{x z}\right)$. The solid squares and solid circles represent the horizontal particle velocity $\left(v_{x}\right)$ and the vertical particle velocity $\left(v_{z}\right)$, respectively. the traction-free boundary condition on the discretized vacuumearth interface. The vertical normal stress may not be zero during the FD calculation for the grid nodes located exactly on the free surface. This generates unsatisfactory results for the simulation of Rayleigh waves.

Here, we propose an improved vacuum formulation to incorporate surface topography and internal discontinuities for FD modeling of Rayleigh waves in the near surface. The proposed method inherits the advantage of conventional vacuum formulation. In addition, the stability of vacuum formulation is improved by an appropriate parameter-averaging scheme in the staggered-grid system. We show that the improved vacuum formulation satisfies the traction-free boundary condition on the vacuum-elastic interface with the consideration of an overlain fictitious layer. The accuracy of the proposed method is benchmarked by comparing the synthetic records with the modeling results of the spectral-element method (SEM) (Komatitsch and Tromp, 1999). We also compare the improved vacuum formulation with the image method. Stability tests of the algorithm are performed by modeling surface waves for earth models including surface topography with Poisson's ratios varying from 0.25 to 0.49 . Finally, we demonstrate the ability to simulate Rayleigh waves for earth models with internal discontinuities.

\section{MODELING OF RAYLEIGH WAVES IN THE 2D P-SV DOMAIN}

The isotropic elastic wave equation in the vertical 2D Cartesian coordinate system can be written in the following velocity-stress form (Virieux, 1986)

$$
\begin{aligned}
& \frac{\partial v_{x}}{\partial t}=b\left(\frac{\partial \tau_{x x}}{\partial x}+\frac{\partial \tau_{x z}}{\partial z}\right), \\
& \frac{\partial v_{z}}{\partial t}=b\left(\frac{\partial \tau_{x z}}{\partial x}+\frac{\partial \tau_{z z}}{\partial z}\right),
\end{aligned}
$$

with the stress-train relations

$$
\begin{gathered}
\frac{\partial \tau_{x x}}{\partial t}=(\lambda+2 \mu) \frac{\partial v_{x}}{\partial x}+\lambda \frac{\partial v_{z}}{\partial z} \\
\frac{\partial \tau_{z z}}{\partial t}=(\lambda+2 \mu) \frac{\partial v_{z}}{\partial z}+\lambda \frac{\partial v_{x}}{\partial x} \\
\frac{\partial \tau_{x z}}{\partial t}=\mu\left(\frac{\partial v_{x}}{\partial z}+\frac{\partial v_{z}}{\partial x}\right)
\end{gathered}
$$

where $\left(v_{x}, v_{z}\right)$ is the particle velocity vector, $b(x, z)$ is the buoyancy (the inverse of density $\rho),\left(\tau_{x x}, \tau_{z z}, \tau_{x z}\right)$ is the stress vector, $\lambda$ and $\mu$ are the Lamé coefficients, and $t$ is the time variable. The model is discretized through the staggered-grid technique to ensure the stability in a heterogeneous medium with large variations of Poisson's ratios. The elastic parameters are shifted as in the scheme shown in Figure 1, where $i$ and $k$ are the indices of the grid node in the $x$-and $z$-directions, respectively. This is equivalent to the $\mathrm{H}$ formulation (Kristek et al., 2002) staggered-grid system in which the vertical particle velocity components are located half a grid position below 
the free surface. Rayleigh waves can be simulated as a part of the solution to the $2 \mathrm{D}$ elastic wave equation.

For a semi-infinite earth model with a planar free surface, the parameters for grid nodes that are close to the free surface can be evaluated by the stress-image technique in second-order accuracy (Graves, 1996). The other edges of the model are usually attached with absorbing boundaries to suppress the spurious reflections caused by the physical truncation of the finite-sized model. The special formulation for free-surface grid nodes introduces difficulties to the modeling in presence of topography. For an earth model with an irregular top surface, the concept of vacuum formulation seems very attractive because of its simplicity of implementation. Unfortunately, simply setting the physical parameters above the free surface to zero does not guarantee the correct generation of Rayleigh waves because the conventional vacuum formulation does not fulfill the traction-free boundary condition ( $\tau_{z z}=\tau_{x z}=0$ at free-surface grid nodes).

\section{THE IMPROVED VACUUM FORMULATION}

\section{Parameter-averaging scheme}

The results of FD modeling can be different depending on the specific choice of staggered-grid configuration. One can locate $v_{x}$ exactly on the free surface, and let $v_{z}$ shift half a grid below it, or vice versa. Both forms of the staggered-grid system have been studied by Kristek et al. (2002), and the differences are usually negligible. On the other hand, a slight modification in the parameteraveraging scheme may yield the distinct stability and accuracy of the modeling (Mittet, 2002). Moczo et al. (2002) chose to use volume harmonic averaging for the shear modulus and volume arithmetic averaging for the density. Mittet (2002) suggested that the averaged rigidity $\bar{\mu}_{x z}$ should be zero if any shear modulus that participates the averaging is zero to ensure the shear stress component $\tau_{x z}$ is always zero on the acoustic-elastic interface. If we consider the shear modulus $\mu_{i, k}$ for the grid node $(i, k)$, the vacuum-elastic interface is similar to the acoustic-elastic interface because $\mu_{i, k}=0$ in both cases. Hence, Mittet's (2002) scheme can be extended following Moczo's (2002) principles of parameter averaging. The expressions for the effective parameters $\bar{b}_{x}, \bar{b}_{z}$, and $\bar{\mu}_{x z}$ are

$$
\begin{gathered}
\bar{b}_{x}= \begin{cases}0, & \text { if } \rho_{\mathrm{i}, \mathrm{k}}=0 \text { and } \rho_{\mathrm{i}+1, \mathrm{k}}=0 \\
\frac{2}{\rho_{i, k}+\rho_{i+1, k}}, & \text { otherwise; }\end{cases} \\
\bar{b}_{z}= \begin{cases}0, & \text { if } \rho_{\mathrm{i}, \mathrm{k}}=0 \text { and } \rho_{\mathrm{i}, \mathrm{k}+1}=0 ; \\
\frac{2}{\rho_{i, k}+\rho_{i, k+1}}, & \text { otherwise; }\end{cases} \\
\bar{\mu}_{x z}=\left\{\begin{array}{cl}
{\left[\frac{1}{4}\left(\frac{1}{\mu_{i, k}}+\frac{1}{\mu_{i+1, k}}+\frac{1}{\mu_{i, k+1}}+\frac{1}{\mu_{i+1, k+1}}\right)\right]^{-1},} & \text { if } \mu_{i, k} \mu_{i+1, k} \mu_{i, k+1} \mu_{i+1, k+1} \neq 0 ; \\
0, & \text { otherwise. }
\end{array}\right.
\end{gathered}
$$

Using the parameter-averaging scheme in equations 6,7 , and 8 is particularly important to ensure the stability of modeling with the vacuum formulation.

\section{Consideration of a fictitious layer}

By applying the proposed parameter-averaging scheme, the vacuum formulation can fulfill the traction-free boundary condition by considering a fictitious layer (shadowed area in Figure 2) above the original topographic model surface. The thickness of this fictitious layer is only half a grid spacing so that the free surface is also shifted half a grid above its original position. In this case, the only stress component located on the free surface is the shear stress component $\tau_{x z}$. The horizontal particle velocity $v_{x}$ and the vertical particle velocity $v_{z}$ are exactly on the free surface after the shift. All the elastic parameters and physical quantities should be set to zero above the free-surface boundary line (the bold line in Figure 2) because they are in the vacuum. The parameters in the original elastic part of the model are left unchanged. According to equation 8 , the effective rigidity $\bar{\mu}_{x z}$ on the free-surface boundary line is always zero if we set the shear modulus $\mu$ to zero for grid nodes in the vacuum. With this strategy, the value of $\tau_{x z}$ is automatically zero during the calculation according to equation 5. The normal stress $\tau_{z z}$ is now under the free surface and located in the elastic part of the model; hence, it does not need to be considered for the freesurface boundary condition.

Although we consider a fictitious layer above the model surface for analysis purposes, no changes are required in the program implementation to explicitly set up this fictitious layer because it is naturally generated by the combination of the staggered-grid form and the proposed parameter-averaging technique. For the shear stress components on the horizontal and vertical surface segments (e.g., points $\mathrm{A}$ and $\mathrm{C}$ in Figure 2) or the inner and outer corners (e.g., points $\mathrm{B}$ and $\mathrm{D}$ in Figure 2), they are always zero due to the averaged zero rigidities. The averaged buoyancy on the freesurface boundary line is twice the buoyancy at the adjacent grid node inside the solid earth. For example, the averaged buoyancy $\bar{b}_{E 0}$ at point $\mathrm{E}_{0}$ (Figure 2) can be calculated by $\bar{b}_{E 0}=$ $2 /\left(\rho_{E-1}+\rho_{E+1}\right)=2 b_{E+1}\left(\rho_{E-1}=0\right.$ in the vacuum $)$, where $\rho_{E-1}$ and $\rho_{E+1}$ are the mass density at grid nodes $\mathrm{E}_{-1}$ and $\mathrm{E}_{+1}$, respectively. Similarly, the averaged buoyancy at point $\mathrm{F}$ is $\bar{b}_{F}=2 b_{E+1}$. This is consistent with the elastic parameter modification scheme in conventional FD modeling with a planar free surface (Mittet, 2002; Xu et al., 2007), which is important to the accuracy of the simulated Rayleigh waves.

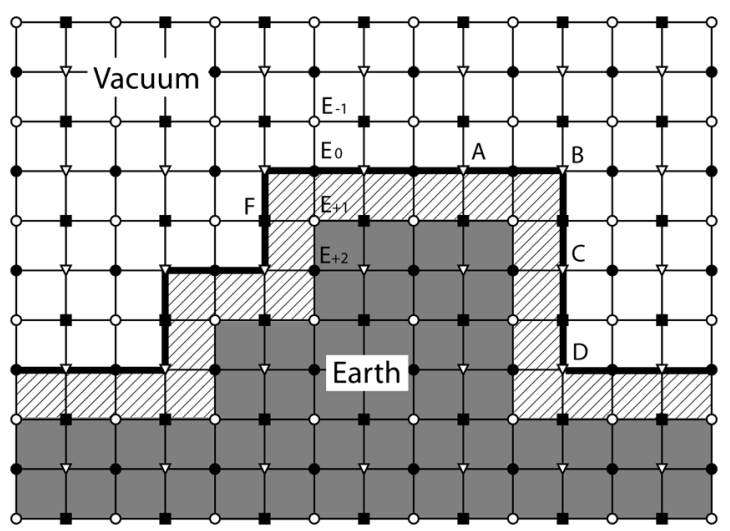

Figure 2. Grid distribution of the improved vacuum formulation in presence of surface topography. The shadowed area is a fictitious layer whose thickness is only half a cell size. The free surface in actual computation is represented by the bold solid line. All parameters above the free surface are set to zero during modeling. The oblique surface segment can be approximated by the staircase shape (e.g., left part of the free surface). 


\section{Velocity updating on the grid nodes}

In the proposed method, the particle velocities should be updated in an averaged scheme because they are not exactly located on the grid nodes in the staggered-grid system. For instance, the vertical particle velocity on the free-surface is calculated by $v_{E+1}=$ $(1 / 2)\left(v_{E 0}+v_{E+2}\right)$, where $v_{E 0}, v_{E+1}$, and $v_{E+2}$ are the vertical particle velocities at points $\mathrm{E}_{0}, \mathrm{E}_{+1}$, and $\mathrm{E}_{+2}$, respectively (Figure 2). This indicates that the vertical particle velocity on the free surface in the proposed vacuum formulation is output as an averaged value of the $v_{z}$ on the fictitious free-surface line and that inside the elastic model. Differing from the stress-image method, the normal stress components on each side of the free surface are not symmetric in the proposed vacuum formation. The particle velocities $v_{x}$ and $v_{z}$ are considered in the elastic part of the model rather than in the vacuum. Because of this, they will not be reset to zero in each time marching loop, which is different from the treatment to the outer corner points in the image method (Robertsson, 1996).

Like in most FD modeling techniques including surface topography, the oblique segments of the topography are approximated by a staircase shape (Hayashi et al., 2001). The primary shortcoming of this approximation is that it needs a fine-grid discretization to reduce the spurious diffractions at the corners of the stairs. In nearsurface seismic modeling focusing on Rayleigh waves, the grid spacing is usually already small enough because a large points per wavelength (ppw) (at least $16 \mathrm{ppw}$ for the shortest-wavelength surface waves) is required to suppress the numerical dispersion of the synthesized Rayleigh waves (Mittet, 2002). In most cases,

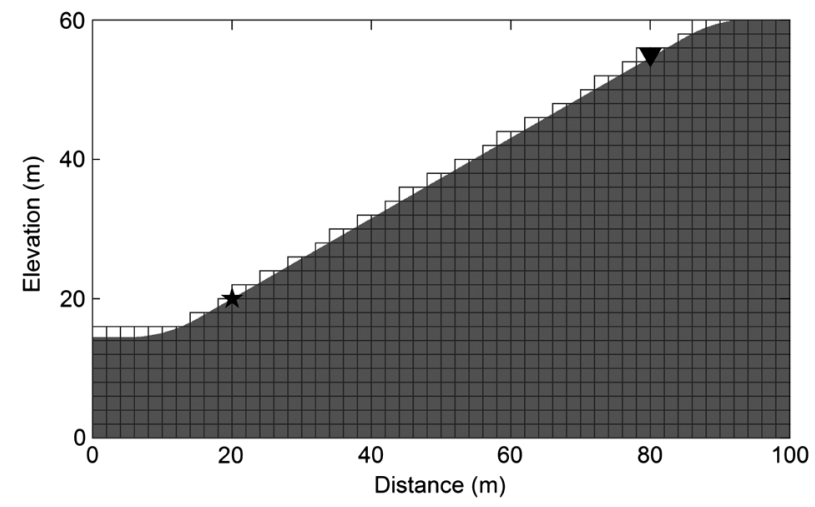

Figure 3. Geometry of the homogeneous slope model for the benchmark tests. The star represents the location of the source. The triangle indicates the location of the receiver. The slope surface is approximated by the staircase shape. The grid lines show the model discretization for FD modeling. The grid spacing illustrated here is only for demonstration. The actual grid spacing $(0.1 \times 0.1 \mathrm{~m})$ during computation is much smaller than shown in this figure. The slope-angles of the free surface in the benchmark are $30^{\circ}, 45^{\circ}$, and $60^{\circ}$.

Table 1. Physical parameters of a homogeneous earth model

\begin{tabular}{ccc}
\hline $\begin{array}{c}\text { P-wave velocity } \\
(\mathrm{m} / \mathrm{s})\end{array}$ & $\begin{array}{c}\text { S-wave velocity } \\
(\mathrm{m} / \mathrm{s})\end{array}$ & $\begin{array}{c}\text { Density } \\
\left(\mathrm{kg} / \mathrm{m}^{3}\right)\end{array}$ \\
\hline 866 & 500 & 2000 \\
\hline
\end{tabular}

the amplitudes of the diffractions are too weak to be noticed on the synthetic records compared to the amplitudes of the direct Rayleigh waves. Hayashi et al. (2001) investigate the diffractions caused by the staircase shape and conclude that the spurious diffractions have less influence on the accuracy of the synthetic record than the numerical dispersion caused by insufficient ppw.

\section{BENCHMARK OF THE ALGORITHM}

In the staircase approximation, an oblique interface is represented by many small horizontal and vertical planar segments. Following the accuracy tests of Robertsson (1996), we use the homogeneous linear-slope models to test the accuracy of the proposed vacuum formulation. In conventional modeling tests, analytical solutions are usually employed to check the accuracy of the results of numerical modeling. However, for a semi-infinite homogeneous elastic medium, the analytical solution to Lamb's problem (Lamb, 1904) only exists when the surface is horizontal. Fortunately, the SEM can accurately approximate the surface topography by using the combination of tetrahedral or hexahedral volume elements. Surface waves can be simulated with high precision by the SEM because the free-surface condition is naturally satisfied regardless of the topography (Komatitsch and Tromp, 1999). Hence, the proposed vacuum formulation can be benchmarked by comparing the trace records with the modeling results for the same model calculated by the SEM.

The geometry of the 2D model used for the benchmark is shown in Figure 3. The physical parameters of the model are listed in Table 1. It is a homogeneous elastic Poisson's solid (Poisson's ratio $\sigma=0.25$ ) with a single oblique flat free surface. The left, right, and bottom edges of the model are attached with the perfectly matched layer (PML) absorbing boundaries (Collino and Tsogka, 2001) during the modeling so that the energy of the spurious reflections can be minimized. All physical parameters above the free surface are set to zero. A vertical vibrating point source and a single receiver are located exactly on the free surface and are separated by a constant horizontal distance of $60 \mathrm{~m}$. The source wavelet is a $20-\mathrm{Hz}$ Ricker wavelet defined by

$$
w(t)=\left[1-2 \pi^{2} f^{2}\left(t-t_{0}\right)^{2}\right] e^{-\pi^{2} f^{2}\left(t-t_{0}\right)^{2}},
$$

where $f$ is the peak frequency, $t$ is the time variable, and $t_{0}$ is the time delay. During the modeling implementation, the source is added to the vertical particle velocity $v_{z}$ on the corresponding grid nodes at each time slice. The left and right sections of the model surface beyond the source and receiver range are rounded to horizontal so as to accommodate the absorbing boundaries and reduce the spurious diffractions at the transition corners. The model was discretized in a fine-grid manner $(0.1 \mathrm{~m} \times 0.1 \mathrm{~m})$ to ensure the stability of the modeling and to reduce the influence of the staircase approximation. We change the slope angle $(\theta)$ of the free surface and run the simulation using the fourth-order staggered-grid FD modeling scheme (second-order in time) described by Graves (1996) with the proposed vacuum formulation. The stability condition is exactly the same as that presented by Graves (1996). Because the source and the receiver are separated constantly in the horizontal direction, the actual offset during the simulations increases with the increment of $\theta$. The source vibrates in the vertical direction for all simulations so that it interacts with the free-surface plane at different angles. Hence, this test simulates the propagation of Rayleigh 
waves under various conditions along different oblique planar surfaces. Figure $4 \mathrm{a}, 4 \mathrm{~b}$, and $4 \mathrm{c}$ demonstrate the synthetic trace records of vertical particle velocity $v_{z}$ when $\theta=30^{\circ}, 45^{\circ}$, and $60^{\circ}$ with the comparison to the SEM results for each corresponding model. All trace records generated by the proposed vacuum formulation agree with the SEM results with negligible differences. This indicates that the P-SV wavefield is accurately simulated using the improved vacuum formulation independent of the angles of slope.

\section{TESTS FOR IRREGULAR SURFACE TOPOGRAPHY}

To confirm the accuracy of the proposed vacuum formulation, an earth model with more complicated surface topography (Figure 5) is employed for the simulation. The model parameters are the same as those in Table 1 . The free surface is composed of a ridge and a valley, which is a typical case for the data acquisition over a small hill in environmental and engineering studies. The 100-channel receiver array is placed on the free surface across the topography. The trace interval is $1 \mathrm{~m}$ in the horizontal direction. The source parameters are the same as those used in the previous benchmark for linear slope models. To minimize the influence of the staircase approximation, the model is discretized into $0.1 \mathrm{~m} \times 0.1 \mathrm{~m}$ cells. Three PMLs are
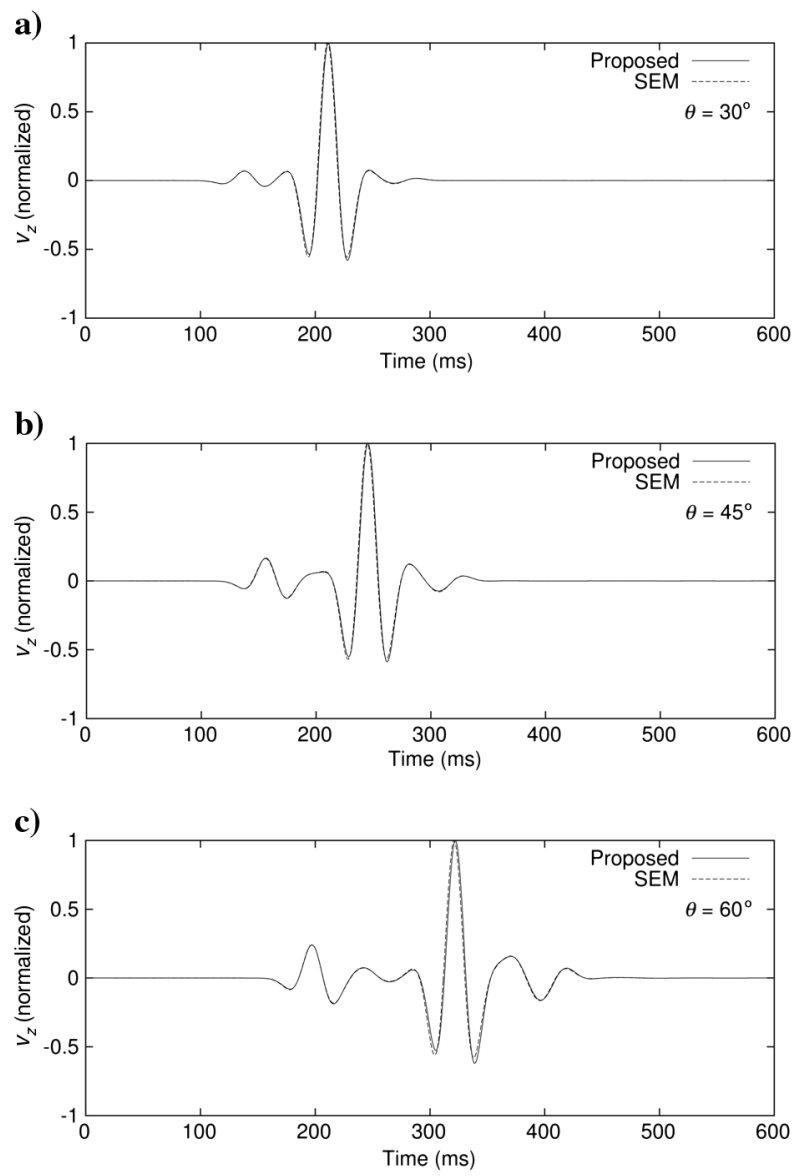

Figure 4. (a) Vertical particle velocities $\left(v_{z}\right)$ recorded for the slope model when the slope-angle (a) $\theta=30^{\circ}$, (b) $\theta=45^{\circ}$, and (c) $\theta=60^{\circ}$. The trace records generated by the proposed vacuum formulation (solid lines) agree well with the overlain SEM results (dashed lines). attached on the left, right, and bottom edges of the model to absorb the spurious reflections. The Courant number defined by

$$
C_{\text {courant }}=\left(\sum \mathrm{c}_{\mathrm{m}}\right) \Delta t v_{\max } \sqrt{\frac{1}{\Delta x^{2}}+\frac{1}{\Delta z^{2}}},
$$

is about 0.3 during the modeling to ensure the stability of the FD algorithm, where $\sum \mathrm{c}_{\mathrm{m}}$ represents the sum of the staggered FD coefficients, $\Delta t$ is the time marching step size, $v_{\max }$ is the maximum $\mathrm{P}$-wave velocity in the model, $\Delta x$ and $\Delta z$ are the horizontal and vertical grid spacing, respectively. The single trace records of $v_{z}$ in Figure $6 \mathrm{a}, 6 \mathrm{~b}$, and $6 \mathrm{c}$ are recorded by the receivers in horizontal offsets of 25,75 , and $100 \mathrm{~m}$. The simulation results from the SEM are used for the trace comparison to check the accuracy of the modeling. The accuracy of the synthetic Rayleigh waves generated with the proposed vacuum formulation is confirmed by good agreement of the $v_{z}$ records with the SEM results.

The image method is also applied to the exact same model to simulate the seismic response in presence of topography for comparison. The trace data (dotted lines in Figure 6a, 6b, and 6c) illustrate that the free surface is correctly implemented because both the amplitude and the arrival time of the Rayleigh waves agree with those generated by the proposed method and the SEM. However, the "tails" after the Rayleigh waves on records generated by the image method are typically characteristic of numerical dispersion (Robertsson, 1996). These numerical errors contaminate the synthetic wavefield and may decrease the accuracy of the dispersion analysis of Rayleigh waves. To suppress the numerical dispersion, the model needs to be discretized into smaller cells so the image method requires more ppw for the simulation of Rayleigh waves. The corresponding time step size also needs to be reduced to ensure the stability of the FD algorithm. This yields a cubic increase of the computing cost with the decrease of grid spacing for a 2D model. According to our tests, the maximum amplitude of the numerical dispersion in the image method can be constrained to no more than $1 \%$ of the original peak amplitude of the Rayleigh waves when the model is discretized into $0.02 \times 0.02 \mathrm{~m}$ cells, which indiates the image method needs approximately five times more ppw than the proposed vacuum formulation for this application. Thus, the proposed vacuum formulation helps reduce the computing cost in FD modeling compared to the image method. For the modeling tests in this paper, we employ a PC cluster with eight quad-core nodes (total 32 logical processors). Each simulation is finished within 26 minutes when using the improved vacuum formulation.

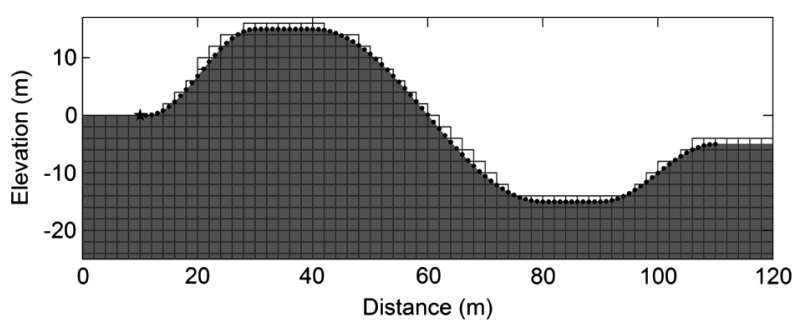

Figure 5. A homogeneous model with the surface topography containing a ridge and a valley. The star represents the location of the source. The solid dots are receivers located on the free surface. The grid lines illustrate the discretization scheme of the model. The grid spacing on this figure is to show concept only. The actual grid spacing for simulations in this paper is much smaller than displayed. 
The test for a same model using the stress-image method takes roughly 10 times the computation time because the ppw has to be increased about five times to get a same accurate result. (Specific results of the time cost comparison may vary with the model size and computing environments such as processor overheads and network latency.)

To test the stability of the proposed vacuum formulation, we increase the P-wave velocity in the previous homogeneous model so that the Poisson's ratio of the model varies from 0.25 to 0.49 and then rerun the modeling. All the simulation results (not shown here) are in good agreement with those computed by SEM, which indicates the proposed method is stable for the tested models with high Poisson's ratios.

Because of the representation of many shallow structures formed by deposition, layered earth models are usually employed in the studies of Rayleigh waves (Xia et al., 2007b; Luo et al., 2009b; Socco et al., 2010). Therefore, we also apply the improved vacuum formulation to a two-layer earth model (Figure 7). This layered earth model has the same surface topography as the previous homogeneous model. We change the physical parameters inside the solid to add another interface beneath the free surface. The geometry of
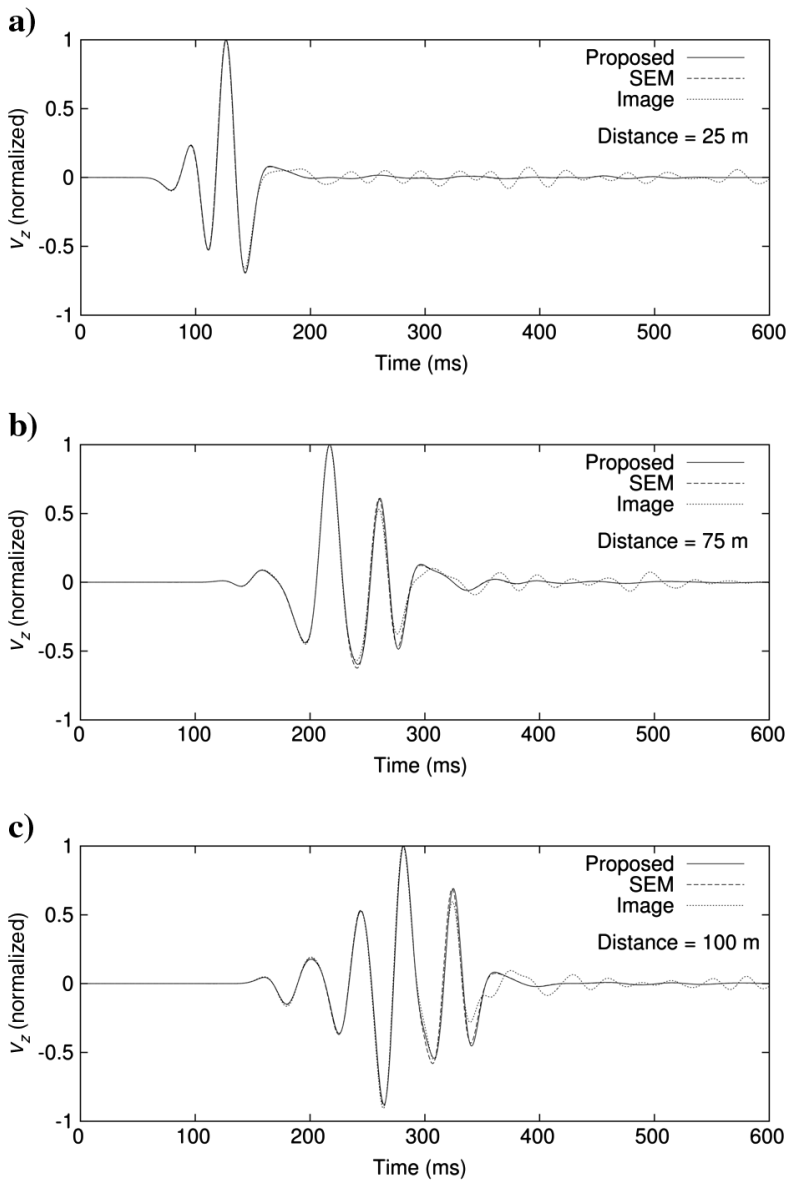

Figure 6. Vertical particle velocities $\left(v_{z}\right)$ recorded in the homogeneous topographic model for the source-receiver distances of (a) $25 \mathrm{~m}$, (b) $75 \mathrm{~m}$, and (c) $100 \mathrm{~m}$. The solid lines are the records generated with the proposed vacuum formulation. The dashed lines are the results from SEM. The dotted lines are trace records simulated with the image method. the internal interface is similar but not identical to the surface topography. This model forms an anticline and a syncline, which is common in the real world. The physical parameters of the top and bottom layers (labeled 1 and 2 in Figure 7) are listed in Table 2. The high Poisson's ratios of the top and bottom layers (about 0.45 and 0.41 , respectively) are commonly seen in the near surface (Xia et al., 2002). All the modeling parameters, such as the grid spacing, source and receiver positions, are exactly the same as those in the test for the model shown in Figure 5. The simplified multiaxial perfectly matched layer (M-PML) technique (Zeng et al., 2011) is used to ensure the stability of absorbing boundaries in this high Poisson's ratio earth model. We run the simulations for the same model using fourth-order FD modeling with the proposed vacuum formulation, the image method, and SEM, respectively. The synthetic trace records of the vertical particle velocities from receiver (horizontal) distances of $25 \mathrm{~m}$ (Figure 8a), $75 \mathrm{~m}$ (Figure 8b), and $100 \mathrm{~m}$ (Figure 8c) are listed for comparisons. The good agreement of the modeling results from the proposed method and SEM illustrates that Rayleigh waves can be simulated along the topographic earth surface for heterogeneous earth models using the proposed vacuum formulation. Similar to the previous homogeneous earth model, there is no notable numerical dispersion on the traces generated by the improved vacuum formulation compared to modeling using the image method (dotted lines in Figure 8a, 8b, and 8c).

\section{INCORPORATION OF INTERNAL DISCONTINUITIES}

When using the proposed vacuum formulation, grid nodes on the topographic free-surface are updated in the exact same manner as internal grid nodes without any special treatments. This allows the internal discontinuities in a model to be incorporated by the same

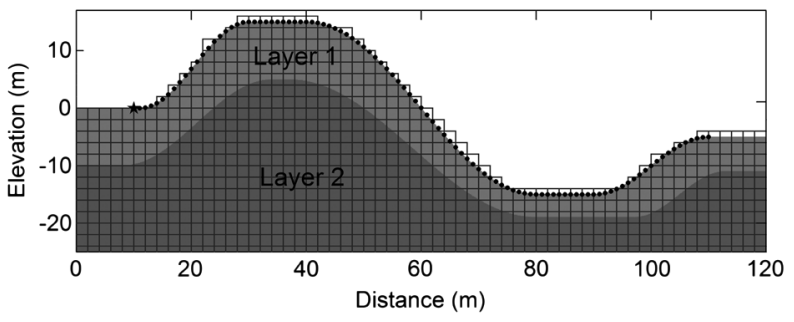

Figure 7. Two-layer earth model with irregular surface topography. The thickness of the top layer varies from 5 to $10 \mathrm{~m}$, thus, the interface geometry between layer 1 and 2 is similar but not equal to the surface topography. The star denotes the source location on the free surface at $(10 \mathrm{~m}, 0 \mathrm{~m})$. The solid dots are receivers on the free surface ranging from the source to $(110 \mathrm{~m},-5 \mathrm{~m})$. The trace interval in the horizontal direction is a constant of $1 \mathrm{~m}$, but varies as measured along the free surface.

Table 2. Physical parameters of a two-layer earth model

\begin{tabular}{lccc}
\hline Layer & $\begin{array}{c}\text { P-wave velocity } \\
(\mathrm{m} / \mathrm{s})\end{array}$ & $\begin{array}{c}\text { S-wave velocity } \\
(\mathrm{m} / \mathrm{s})\end{array}$ & $\begin{array}{c}\text { Density } \\
\left(\mathrm{kg} / \mathrm{m}^{3}\right)\end{array}$ \\
\hline 1 & 1600 & 500 & 1800 \\
2 & 2000 & 800 & 2000 \\
\hline
\end{tabular}


algorithm as the surface topography. Near-surface earth models that contain internal discontinuities such as underground tunnels and shallow cavities have been investigated in many environmental and engineering studies (Xia et al., 2004, 2007a; Gelis et al., 2005). Shallow tunnels are often man-made and angular. The wall of a tunnel can be considered as a high contrast discontinuous interface for the propagation of seismic waves. Figure 9 shows the staggeredgrid system with the proposed vacuum formulation for a model with a rectangular void inside an elastic medium. The interior of the void can be considered a vacuum during the modeling because the density of air is much less than that of the surrounding materials. The four edges of the void are free-surface boundaries and need special treatment for FD modeling if using the image method. In contrast, when utilizing the proposed vacuum formulation, they are naturally incorporated as the internal interfaces with no extra operations.

Figure 10 displays the synthetic shot gather generated by the fourth-order FD modeling with the proposed vacuum formulation for a rectangular tunnel inside a $2 \mathrm{D}$ homogeneous half-space earth model. The tunnel is $10 \times 10 \mathrm{~m}$ with its top at a depth of $20 \mathrm{~m}$. The center of the tunnel is located exactly in the middle of the 100-channel receiver array. The nearest offset and the receiver
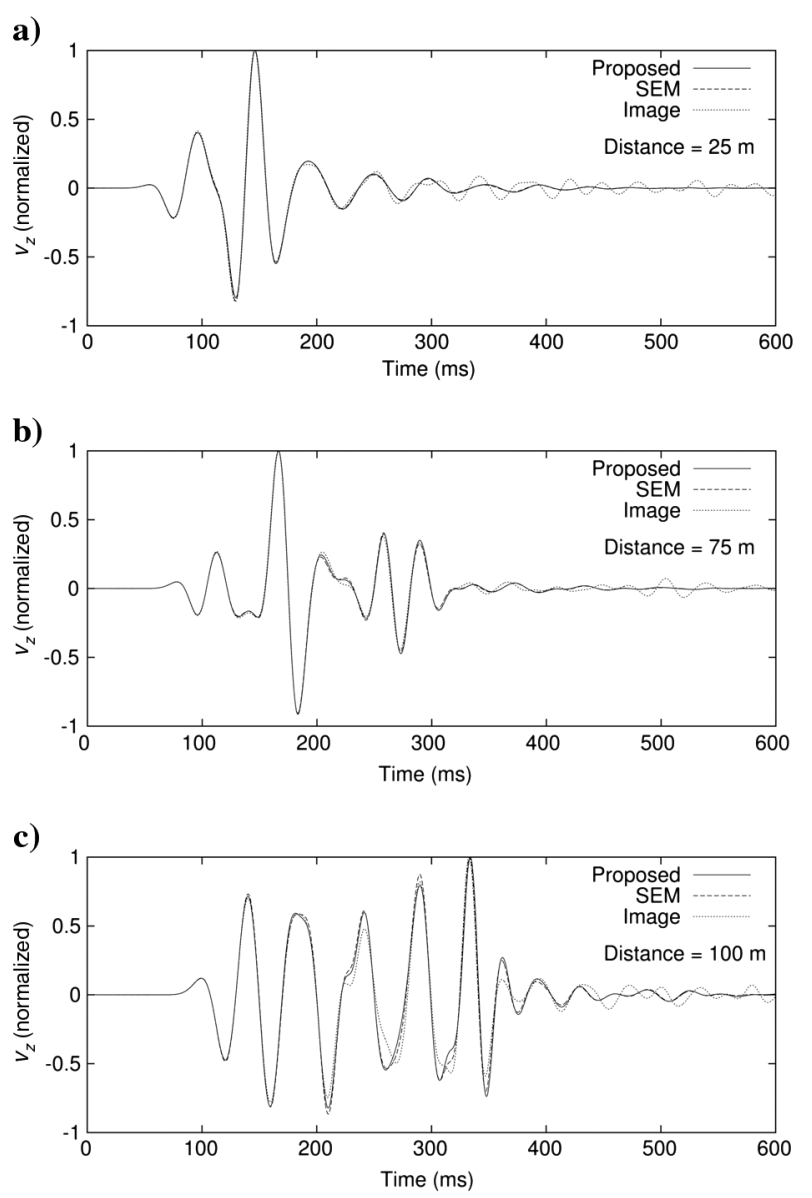

Figure 8. Vertical particle velocities $\left(v_{z}\right)$ recorded in the two-layer topographic earth model for the source-receiver distances of (a) $25 \mathrm{~m}$, (b) $75 \mathrm{~m}$, and (c) $100 \mathrm{~m}$. The solid lines are the records generated with the proposed vacuum formulation. The dashed lines are the results from SEM. The dotted lines are results generated by the image method. interval are both $1 \mathrm{~m}$. To increase the resolution of the seismogram, we use a $50-\mathrm{Hz}$ Ricker wavelet. The source excites vertically on the free surface to generate strong Rayleigh waves. The P-wave velocity, the S-wave velocity, and the mass density of the half-space are listed in Table 1. The PML absorbing technique is applied to the left, right, and bottom edges of the model. The Rayleigh-wave diffractions from the corners of the tunnel are observed on the synthetic record. The wavefronts of the diffracted waves are clearly shown in the wavefield snapshot at time $t=150 \mathrm{~ms}$ (Figure 11). The arrival time of the diffracted Rayleigh waves on each trace agrees with the theoretical value obtained from the traveltime

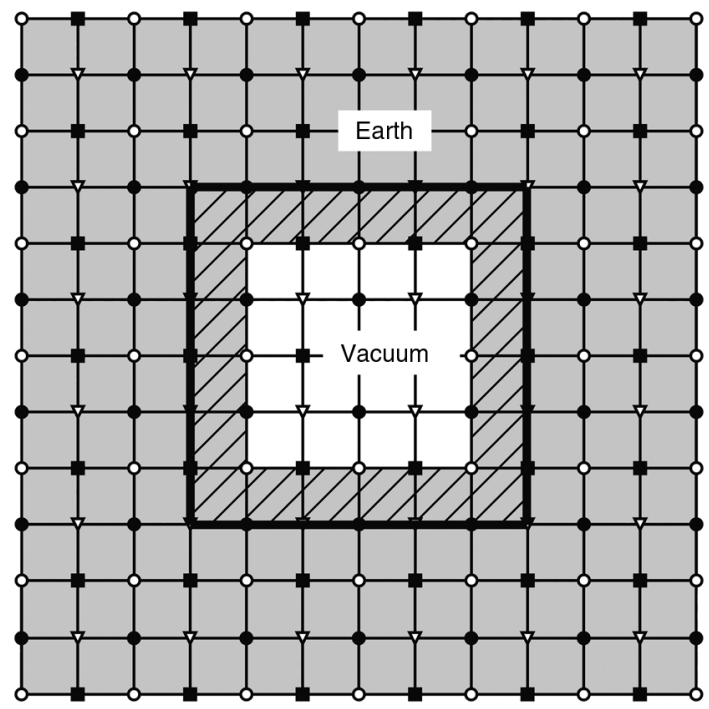

Figure 9. Grid distribution for a model containing an internal discontinuity. The internal vacuum-elastic interface is attached with a fictitious layer (the shadowed area) so that the boundary of the internal discontinuities can be incorporated using the same algorithm at the top surface.

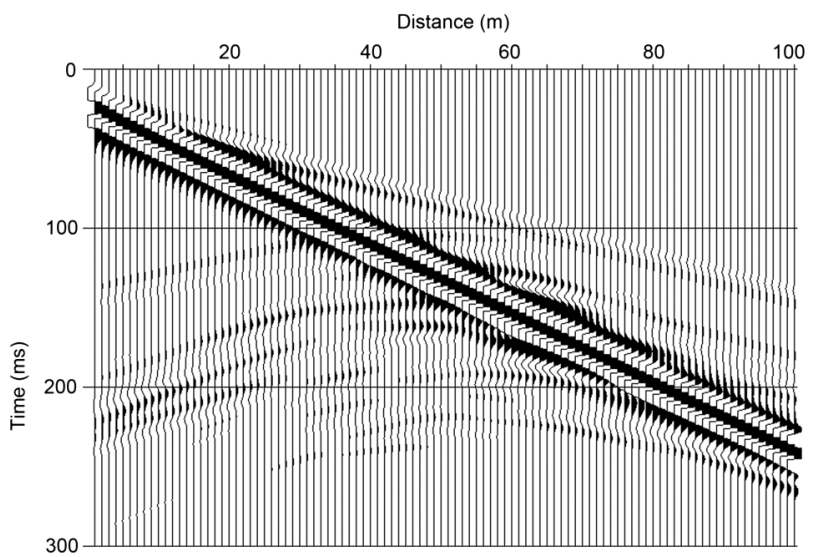

Figure 10. A synthetic shot gather generated for the tunnel model in Figure 10 by fourth-order FD modeling with the improved vacuum formulation. Diffractions are seen on the seismogram at distances from 5 to $50 \mathrm{~m}$ in the time window of between 100 and $250 \mathrm{~ms}$. A large gain factor is applied to enhance the visibility of the diffracted events. 


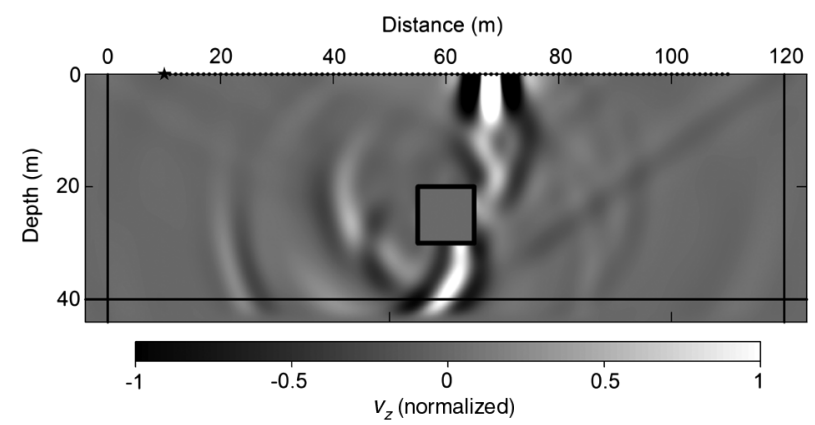

Figure 11. A wavefield snapshot at $150 \mathrm{~ms}$ of the vertical particle velocities $\left(v_{z}\right)$. The square represents the tunnel. The solid lines are the interfaces of PML absorbing boundaries. The star denotes the source on the free surface and the dots denote the 100-channel receiver array stretching over the tunnel. The wavefronts of the diffractions are visible on the snapshot.
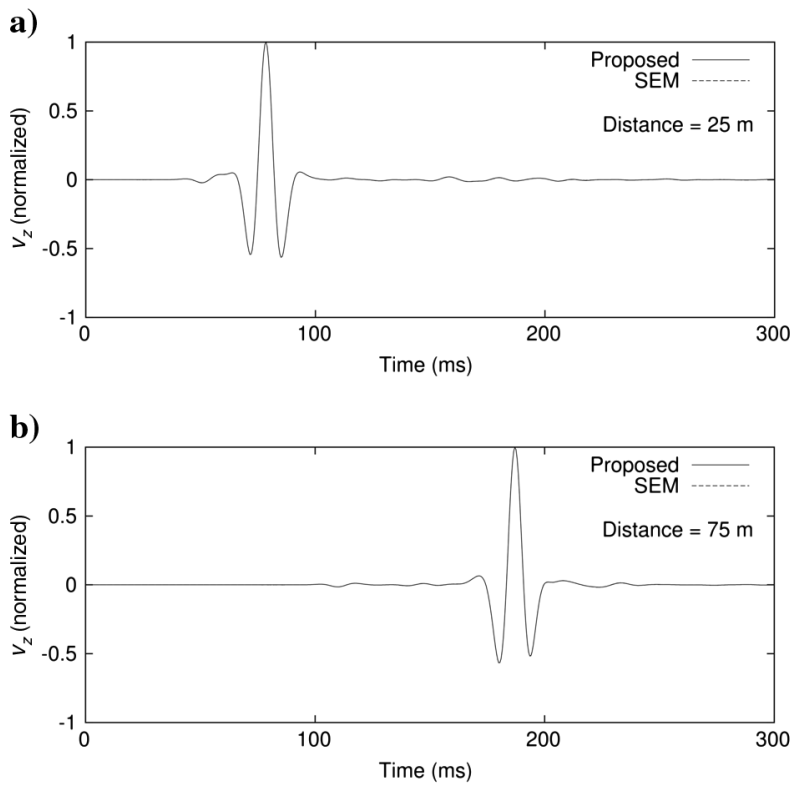

Figure 12. Trace record at distance of (a) $25 \mathrm{~m}$, and (b) $75 \mathrm{~m}$ that extracted from the synthetic shot gather shown in Figure 11 (solid lines) compared to the SEM results (dashed lines).

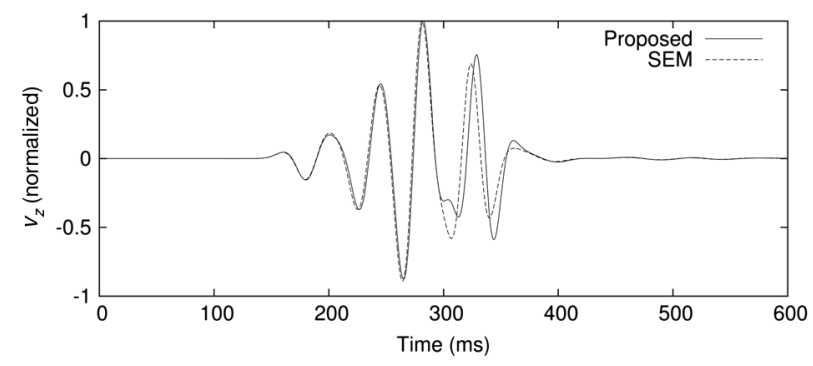

Figure 13. The FD computed synthetic trace (solid line) for the model shown in Figure 5 but using only 32 ppw for the proposed method. The SEM result is shown as a dashed line for comparison. equation developed by Xia et al. (2007a). Detailed single-trace comparisons (e.g., the traces at the distances of 25 and $75 \mathrm{~m}$ ) with the modeling results of the SEM in Figure 12a and $12 \mathrm{~b}$ confirm that the $\mathrm{P}-\mathrm{SV}$-wavefield is correctly simulated by the proposed vacuum formulation for the earth model containing internal discontinuities.

\section{DISCUSSION}

The ppw used for most simulation tests in this paper is above 90 , which is much greater than the case of conventional modeling with planar free-surface (16 ppw, [Mittet, 2002]). More tests show that, when a model contains only horizontal and vertical free surfaces (e.g., the slope model in the benchmark section when $\theta=0^{\circ}$ and $\theta=90^{\circ}$ ), 16 ppw is sufficient to generate accurate Rayleigh waves with no significant numerical dispersion. When there is an oblique surface, the ppw requirement increases with the slope angle. This is related to representing the free-surface topography with the discretized grids in FD modeling. Figure 13 shows a synthetic trace computed from the same model shown in Figure 5 at the horizontal offset of $100 \mathrm{~m}$ but using $32 \mathrm{ppw}$ with the proposed method. The wavelets on the FD simulated trace are delayed compared to the SEM result, which decreases the phase velocities of the simulated high-frequency Rayleigh waves. This delay is also a typical numerical error due to insufficient ppw (Dablain, 1986).

Although the proposed vacuum formulation requires stricter ppw conditions than conventional modeling with the horizontal planar free surface, the requirement is still reasonable for many nearsurface studies because an irregular interface needs to be discretized in a fairly fine-grid manner for a proper approximation to suppress the spurious diffractions caused by the staircase corners. The grid spacing after discretization for a practical simulation usually yields more ppw than the minimum requirement. For example, Gelis et al. (2005) used over $30 \mathrm{ppw}$ during their numerical investigation for shallow cavities, which is already about twice the conventional critical requirement (16 ppw). Compared with the image method, the improved vacuum formulation requires fewer ppw and would not significantly increase the computing cost in practice.

\section{CONCLUSIONS}

The conventional vacuum formulation can be improved to satisfy the traction-free boundary condition on the vacuum-elastic interface to simulate accurate Rayleigh waves using fourth-order FD modeling in the presence of surface topography. In the improved vacuum formulation, parameters at the surface grid nodes are averaged using the same scheme used for the internal grid nodes. The free surface is shifted half a grid spacing so that only the shear stress component needs to be considered to fulfill the free-surface boundary condition. By applying arithmetic averaging for the density and harmonic average for the shear modulus on the vacuum-elastic interface, the free-surface boundary condition is naturally satisfied in a staggeredgrid system. Grid nodes on the free surface are updated in the same manner as internal grid nodes without any special treatments. Compared to the image method, the improved vacuum formulation is easier to implement and needs fewer ppw during the modeling to generate accurate Rayleigh waves without notable numerical dispersion. It is stable for the homogeneous and heterogeneous models tested in this study that contain irregular surface topography and internal discontinuities with Poisson's ratios ranging from 0.25 to 0.49 . Rayleigh waves can be accurately simulated along the topographic earth surface using the improved vacuum formulation 
with fourth-order staggered-grid FD modeling. The proposed vacuum formulation can be easily implemented in existing 2D FD modeling codes.

\section{ACKNOWLEDGMENTS}

The authors thank Koichi Hayashi for sharing his modeling data for reference. We thank associate editor Jeff Shragge, reviewer Sjoerd de Ridder, and two other anonymous reviewers for their constructive suggestions and critiques that significantly improved the quality of the manuscript. We also thank Mary Brohammer for editing the manuscript.

\section{REFERENCES}

Aki, K., and P. G. Richards, 2002, Quantitative seismology, 2nd ed.: University Science Books.

Calderón-Macías, C., and B. Luke, 2007, Improved parameterization to invert Rayleigh-wave data for shallow profiles containing stiff inclusions: Geophysics, 72, no. 1, U1-U10, doi: 10.1190/1.2374854.

Carcione, J. M., 1992, Modeling anelastic singular surface waves in the earth: Geophysics, 57, 781-792, doi: 10.1190/1.1443292.

Collino, F., and C. Tsogka, 2001, Application of the PML absorbing layer model to the linear elastodynamic problem in anisotropic heterogeneous media: Geophysics, 66, 294-307, doi: 10.1190/1.1444908.

Dablain, M. A., 1986, The application of high-order differencing to the scalar wave equation: Geophysics, 51, 54-66, doi: 10.1190/1.1442040.

Gelis, C., D. Leparoux, J. Virieux, A. Bitri, S. Operto, and G. Grandjean, 2005, Numerical modeling of surface waves over shallow cavities: Journa of Environmental and Engineering Geophysics, 10, 111-121, doi: 10.2113/JEEG10.2.111.

Graves, R. W., 1996, Simulating seismic wave propagation in 3D elastic media using staggered-grid finite differences: Bulletin of Seismological Society of America, 86, 1091-1106.

Hayashi, K., D. R. Burns, and M. N. Toksöz, 2001, Discontinuous-grid finite-difference seismic modeling including surface topography: Bulletin of Seismological Society of America, 91, 1750-1764, doi: 10.1785/ 0120000024 .

Jih, R. S., K. L. McLaughlin, and Z. A. Der, 1988, Free-boundary conditions of arbitrary polygonal topography in a two-dimensional explicit elastic finite-difference scheme: Geophysics, 53, 1045-1055, doi: 10.1190/ 1.1442541

Komatitsch, D., and J. Tromp, 1999, Introduction to the spectral-element method for 3-D seismic wave propagation: Geophysical Journal International, 139, 806-822, doi: 10.1046/j.1365-246x.1999.00967.x.

Kristek, J., P. Moczo, and R. J. Archuleta, 2002, Efficient methods to simulate planar free surface in the 3D 4th-order staggered-grid finite-difference schemes: Studia Geophysica et Geodaetica, 46, 355-381, doi: 10.1023/ A:1019866422821.

Lamb, H., 1904, On the propagation of tremors over the surface of an elastic solid: Philosophical Transactions of the Royal Society A: Mathematical, Physical and Engineering Sciences, 203, 1-42, doi: 10.1098/rsta.1904 .0013 .

Levander, A. R., 1988, Fourth-order finite-difference P-SV seismograms: Geophysics, 53, 1425-1436, doi: 10.1190/1.1442422.

Luo, Y., J. Xia, R. D. Miller, Y. Xu, J. Liu, and Q. Liu, 2009a, Rayleighwave mode separation by high-resolution linear Radon transform: Geophysical Journal International, 179, 254-264, doi: 10.1111/gji.2009.179 issue-1.
Luo, Y., J. Xia, Y. Xu, C. Zeng, R. D. Miller, and Q. Liu, 2009b, Dippinginterface mapping using mode-separated Rayleigh waves: Pure and Applied Geophysics, 166, 353-374, doi: 10.1007/s00024-009-0451-z.

Mittet, R., 2002, Free-surface boundary conditions for elastic staggeredgrid modeling schemes: Geophysics, 67, 1616-1623, doi: 10.1190/ 1.1512752 .

Moczo, P., J. Kristek, V. Vavryčuk, R. J. Archuleta, and L. Halada, 2002, 3D heterogeneous staggered-grid finite-difference modeling of seismic motion with volume harmonic and arithmetic averaging of elastic moduli and densities: Bulletin of Seismological Society of America, 92, 30423066, doi: $10.1785 / 0120010167$.

Nazarian, S., and K. H. Stokoe II, 1984, In situ shear wave velocities from spectral analysis of surface waves: 8th Conference on Earthquake Engineering, 3, 31-39.

Robertsson, J. O. A., 1996, A numerical free-surface condition for elastic/ viscoelastic finite-difference modeling in the presence of topography: Geophysics, 61, 1921-1934, doi: 10.1190/1.1444107.

Socco, L. V., S. Foti, and D. Boiero, 2010, Surface-wave analysis for building near-surface velocity models - Established approaches and new perspectives: Geophysics, 75, no. 5, A83-A102, doi: 10.1190/1.3479491.

Tessmer, E., D. Kosloff, and A. Behle, 1992, Elastic wave propagation simulation in the presence of surface topography: Geophysical Journal International, 108, 621-632, doi: 10.1111/gji.1992.108.issue-2.

Virieux, J., 1986, P-SV wave propagation in heterogeneous media: Velocitystress finite-difference method: Geophysics, 51, 889-901, doi: 10.1190/ 1.1442147.

Xia, J., C. Chen, P. H. Li, and M. J. Lewis, 2004, Delineation of a collapse feature in a noisy environment using a multichannel surface wave technique: Geotechnique, 54, 17-27, doi: 10.1680/geot.2004.54.1.17.

Xia, J., R. D. Miller, and C. B. Park, 1999, Estimation of near-surface shear-wave velocity by inversion of Rayleigh waves: Geophysics, 64 691-700, doi: 10.1190/1.1444578.

Xia, J., R. D. Miller, C. B. Park, J. A. Hunter, J. B. Harris, and J. Ivanov, 2002 , Comparing shear-wave velocity profiles inverted from multichannel surface wave with borehole measurements: Soil Dynamics and Earthquake Engineering, 22, 181-190, doi: 10.1016/S0267-7261(02) 00008-8.

Xia, J., R. D. Miller, C. B. Park, and G. Tian, 2003, Inversion of high frequency surface waves with fundamental and higher modes: Journal of Applied Geophysics, 52, 45-57, doi: 10.1016/S0926-9851(02) 00239-2.

Xia, J., J. E. Nyquist, Y. Xu, M. J. S. Roth, and R. D. Miller, 2007a, Feasibility of detecting near-surface feature with Rayleigh-wave diffraction: Journal of Applied Geophysics, 62, 244-253, doi: 10.1016/j.jappgeo .2006.12.002.

Xia, J., Y. Xu, and R. D. Miller, 2007b, Generating image of dispersive energy by frequency decomposition and slant stacking: Pure and Applied Geophysics, 164, 941-956, doi: 10.1007/s00024-007-0204-9.

Xia, J., Y. Xu, R. D. Miller, and C. Chen, 2006, Estimation of elastic moduli in a compressible Gibson half-space by inverting Rayleigh wave phase velocity: Surveys in Geophysics, 27, 1-17, doi: 10.1007/s10712-005$7261-3$

Xu, Y., J. Xia, and R. D. Miller, 2007, Numerical investigation of implementation of air-earth boundary by acoustic-elastic boundary approach: Geophysics, 72, no. 5, SM147-SM153, doi: 10.1190/1.2753831.

Zahradník, J., P. Moczo, and F. Hron, 1993, Testing four elastic finitedifference schemes for behavior at discontinuities: Bulletin of Seismological Society of America, 83, 107-129.

Zeng, C., J. Xia, R. D. Miller, and G. P. Tsoflias, 2011, Application of the multiaxial perfectly matched layer to near-surface seismic modeling with Rayleigh waves: Geophysics, 76, no. 3, T43-T52, doi: 10.1190/ 1.3560019 . 